N. E. Piskunov, W. W. Weiss, D. F. Gray, eds.

\title{
Round Table Summary: Winds and Pulsating Stars
}

\author{
Lee Anne Willson \\ Department of Physics and Astronomy, Iowa State University, Ames IA \\ 50011, USA
}

\begin{abstract}
Speakers for this session were selected according to our perceptions about the frontier areas in dynamical atmospheres, the atmospheres of very cool stars, and stellar winds. This summary of posters and topics raised in the round table discussion opens with a brief summary of current issues in modeling pulsating stars and winds. Observational constraints are emphasized in Section 3. on "peeling the onion" of the atmosphere/wind structure. Section 4. covers a variety of methods for detecting and measuring stellar winds. The concluding remarks include some questions facing theorists and observers working in this field.
\end{abstract}

\section{Introduction}

Susanne Höfner and I were asked to select speakers for this session, first called "Cool stars and stellar winds" and later made more general. We identified the "frontier areas" listed below, and sought, in our choice of speakers and assignments to each, to span as much as we could of this list.

Frontier areas in dynamical atmospheres and winds:

1. Non-LTE effects in dynamical atmospheres, including instabilities associated with molecules or dust.

2. Departures from spherical symmetry and/or steady outflow.

3. Synthesizing observable quantities - including, but not limited to, spectrum synthesis.

4. Interaction of winds with companion objects - stars or planets.

Item 4 did not end up being covered in the session, although the posters by Linsky et al. and Wood et al. introduced an interesting variation by detecting a line feature ascribed to the interaction of the wind with the ambient interstellar medium, and some of the posters in the "departure from spherical symmetry" session were also relevant (e.g. Struck et al., D26). We asked Peter Woitke to give the keynote talk on topic 1 . His review neatly summarizes a variety of ongoing efforts and highlights a number of the most urgent challenges. For item 2 and the observational take on item 1 we invited two speakers: Gerard van Belle to tell us about opportunities emerging from stellar interferometry, and Elizabeth Humphreys to present results of maser observations in and around red 
giants (mainly Mira variables). Note that there is another round table discussion covering interferometry in this volume, so our coverage will be brief. Finally, I prevailed on Susanne Höfner to review topic 3 - a decision well-supported by the observation that more than half of the posters submitted for this session had her as a co-author. The round table discussion on Friday, June 20, included our four review speakers plus the authors of four of the poster papers: Lebzelter, Wood, Aringer, and Josselin.

After reviewing the topics presented via posters as well as the contents of the review papers, and considering elements of the discussion at the round table, I have chosen to bin the results in this essay into three themes:

I Modeling: What is the essential physics?

II Peeling the onion: What is the structure of the atmosphere and wind?

III Winds: How to model, how to measure?

The final section lists three questions currently challenging theorists and observers. As noted in other round table discussions, this summary makes no pretense of being a complete review of the subject, and its content includes more idiosyncratic comment and speculation than a typical paper or review. The reader is referred to other sources - such as the companion reviews on hot star and cool star winds in ARA\&A volume 38 (Kudritzki \& Puls 2000, Willson 2000) as well as the reviews in this volume - for more complete and balanced reviews.

\section{Frontiers in the modeling of pulsating atmospheres and stellar winds.}

As ably described in Woitke's review, a fully satisfactory model for a dynamical cool-star atmosphere with associated wind would include the coupling of the atmosphere to the interior (pulsation or convection), fully non-LTE treatment at densities below photospheric, detailed (non-equilibrium, time-dependent) chemistry of molecules and dust, multi-fluid treatment of the dust/molecule/atomic gas components in the wind, and perhaps also the generation and effects of magnetic fields. The models should allow for phenomena occurring on a range of timescales from short period fluctuations and fast processes to (for red giants) dynamical and thermal timescales lasting years to centuries. Instabilities (on a range of spatial scales) should be allowed for and permitted to grow. Clearly implied by both the convection case and the magnetic field component are that these models should be fully three-dimensional, and instabilities also tend to produce departures from spherical symmetry. Sufficient information should be retained to allow a full spectrum synthesis for each model. Quite clearly, we are very far from this ideal (and it may never be sensible to try for all these features in a single model). So, for every purpose for which models are constructed, choices have to be made about both which processes to include and to what detail to include them.

Broadly, there are now two general classes of models being computed. The first approach, represented by the Bowen models (1988, Bowen \& Willson 1991, 
Bowen 2003 in prep.), attempt to provide an adequate approximation to a range of phenomena likely to be important, and then study the balance and interaction among these processes. The second approach, used by most of the other groups whose work Woitke reviewed for us, is to identify processes that are likely to be important, and to push for a relatively detailed inclusion of those processes. With enough experience, it should ultimately be possible to use the results of the first kind to inform the choices in the second type, and the results of the second kind to inform the approximations used for the first type. The study reviewed by Höfner concerning how many frequency bins are needed to get an adequate representation of the radiation field for the atmospheric structure calculations is a fine example of how symbiosis may be carried forward.

How good are current models? Bowen's models do a remarkable job of matching the range of stellar parameters over which the final "superwind" mass loss occurs, in spite of their relative simplicity. However, they do not contain the kind of information or detailed physics needed to provide the input for spectrum synthesis modeling, and there is no easy way to use them for this purpose because the non-LTE approximation needs to be matched in the radiative transfer and it is difficult to take a relaxation-rate approximation and match it with detailed NLTE (while the reverse process is much simpler). The models presented here by Höfner (review) and Aringer et al. (poster) as well as others described below are remarkably successful at modeling some parts of the stellar spectra, but still give anomalous results particularly for some molecular features. These classes of models also have yet to produce a mass loss law that results in a good match with the termination of the AGB as constrained by a variety of observations (Willson 2000).

It is an interesting puzzle: Why do the Bowen models do so much better at matching the observations of the collective properties of the stars at the tip of the AGB? Perhaps the answer lies in this: By constraining the models to follow reasonable, internally consistent, evolutionary tracks, scaling the driving to the luminosity, and making simple consistent approximations to all the key processes, Bowen's models reveal the dependence of the mass loss rate on stellar parameters and hence tell us which stars are losing mass at a rate sufficient to end their evolution. With slightly different choices of parameters such as the mixing length used to select the family of evolutionary tracks, or the thermal recovery timescale, or of the dust formation conditions, or the ratio of pulsation driving to $L$, the pattern of mass loss shifts only slightly towards higher or lower $L$ for a given progenitor $M$. Thus the utility of these grids comes in part because they reveal the major trends very well, while the details may or may not be accurate for a given $M, L, R$ and $Z$.

The other classes of models have been computed for more limited grids and without some of the model-to-model constraints of Bowen's most recent grids. However, more detailed treatment of radiative transfer gives a photospheric structure that may be checked against spectra or broadband spectral energy distributions because the source function is more easily derived from the dynamical calculations. Without the global constraints used by Bowen, and including more details of some processes, this second class of models may reveal (real or not) variations in the dependence of mass loss rates on stellar parameters that apply over a relatively narrow range of stellar parameters, and may thus 
not reveal as readily the global trends. It is also worth a reminder at this point that the situation is further complicated by the fact that the Bowen models concern oxygen-rich Miras while most of the other modeling groups have focused on carbon star models, where some of the details of the chemistry and grain formation are more readily managed. It may very well be true that the mass loss function for carbon stars is significantly different from that for oxygen-rich stars.

The poster by Aringer et al. described their use of MARCS hydrostatic models and also non-grey dynamical models to compute molecular line spectra as a function of stellar parameters. Their goal is to reverse the process and use spectra to determine stellar effective temperatures and gravities, a classic problem in stellar atmospheres but one made challenging by the character of molecular spectra for the coolest stars - millions of lines are needed and as lines are the dominant source of opacity, there is no conventional continuum in these stars. The results are encouraging for stars with relatively weak water absorption and effective temperatures above $3000 \mathrm{~K}$, but difficulties are encountered for the apparently coolest stars and the variable stars. While they prudently stopped short of suggesting a reason for this difficulty, it is tempting to speculate that the departures from equilibrium expected at relatively high altitudes in variable stars of all types could lead to very different temperature stratification than in the MARCS results (with, also, severe departures from LTE making temperature no longer a simple single parameter).

Hron et al. presented results of comparing synthetic spectra with ISO spectra of two carbon stars with estimated mass loss rates around $2 \times 10^{-6} M_{\odot} /$ year - very much in the interesting range for AGB evolution. Equilibrium chemistry was used. The results are in good qualitative agreement although there are systematic differences; these may perhaps be decreased by fine-tuning model parameters or they may ultimately require fundamental improvements in the theoretical models.

Once dust forms in the atmosphere of a star, it will begin to move relative to the gas. There is a complex feedback situation between grain size and differential speed: The relative velocity depends on the size, but the growth or destruction also depends on the relative velocity. In addition, the gas experiences compression with heating in shocks, and grain growth depends on both temperature and density. Depending on how these processes are handled, various interesting instabilities can occur. The poster by Sandin and Höfner includes a detailed look at some of these processes, although necessarily still with mostly equilibrium chemistry.

Final note: There is a tendency to try to use the models to set up clear dichotomies - e.g. AGB mass loss is either dust driven or pulsation driven. For a given choice of stellar parameters, a model with dust will typically have orders of magnitude more mass loss than one without, prompting the conclusion that dust must play a pivotal role. However, models with slightly higher luminosities or lower masses can be constructed where a comparable mass loss rate is produced without dust. For a given star, presumably, either a dusty wind or a non-dusty wind will terminate the AGB. However, for a steep dependence of the mass loss rate on stellar parameters, as appears to be needed to produce the "superwind" phase, for a given set of stellar parameters a small change in the model will 
lead to a big change in $\dot{M}$ (e.g. dust $\dot{M} \gg$ non-dust) but a small change in stellar parameters can lead to a compensatingly large change in $\dot{M}$ (the cliff doesn't move far for without dust vs. with dust). So the final $L$ on the AGB for stars never able to make dust (low mass and/or low $Z$ ) may not be very much different from the final $L$ for stars that do make dust. I won't be surprised if that the general result for AGB evolution (at this $L$, stars of initial mass $M$ and composition $Z$ will lose their envelopes and "die") is well determined long before the details (this star of this $L, R, M, Z$ has this mass loss rate) are sorted out. (For a more extensive discussion, see Willson 2000 and references therein.)

\section{Peeling the onion: What is the structure of the atmosphere and wind?}

Traditional stellar atmosphere models are static, with all processes at or very near equilibrium. LTE generally describes the conditions where most of the spectrum is formed, near the photosphere. Under these conditions, with nonpathological opacities, conditions vary gently from location to location in readily computed gradients, and there is often a nearly one-dimensional correlation between effective temperature and the appearance of the spectrum over a wide range of frequencies. In the models for dynamic atmosphere for cool stars with winds, conditions from one position to another in the atmosphere may be sensitive to details of the model and/or time-dependent. Instabilities may create horizontal/azimuthal variations as well as temporal ones. Fortunately, new tools are emerging that will let us "peel the onion" much more directly than has ever been possible.

Many mass-losing, cool stars are the source of maser emission from $\mathrm{SiO}$, $\mathrm{H}_{2} \mathrm{O}$, and $\mathrm{OH}$ - with the larger amplitude pulsators typically being the most enthusiastic sources of maser emission. $\mathrm{SiO}$ masers form close to the star, $\mathrm{H}_{2} \mathrm{O}$ and $\mathrm{OH}$ masers farther out, giving good radial resolution once the mechanisms are clear. Humphreys, in her review, notes that the dominant $\mathrm{SiO}$ emission is from a ring around the star - i.e. tangential amplification is most effective. The pattern seen in VLBA studies suggests clumpy structure in the outer atmosphere or wind, and cyclical variations consistent with a rising shock front have been seen in at least one case. Finally, for TX Cam, there is a second smaller ring of polarization (the diamond on the ring?) seen in some of the $\mathrm{SiO}$ observations reviewed by Humphreys; I will bravely speculate that this feature is the result of a planet or small companion orbiting in the wind of the red giant, as the time-variation of this feature is consistent also with orbital motion.

Gerard van Belle ably reviewed another new tool: Optical interferometry is now joining radio VLBI in allowing us to resolve at least the largest stars and study their size, if not eventually also their appearance, as a function of time and wavelength. While the topic of interferometry is covered elsewhere in this volume, his central message deserves repeating: We have opportunities for some revealing new information, but the interferometrists need help from the models in determining what observations will be most useful as well as for interpreting the observations after they have been made.

A significant step towards improved interpretation of observations was presented in the poster by Lebzelter et al: Synthetic line profile variations for CO in 
a pulsating star model show clearly that there is no need to invoke a "stationary layer" to explain the strong absorption near $v=0$ that is observed. This is very good news as the physics of creating and supporting a "stationary layer" in an outflow is very far from obvious (and probably not possible). The model cited in this paper was also used in the paper by Hron et al. to fit the ISO spectrum of S Cep, and further invoked in the poster by Nowotny et al. on atmospheric kinematics.

Začs et al. described a long-term observational study of pulsating carbon stars showing that while at maximum light $\mathrm{C}_{2}$ absorption is seen - as is expected for such cool stars - near minimum the spectra are dramatically different. In RW LMi the spectrum near $5500 \AA$ is nearly featureless at minimum, with only strong emission lines including [O I]. They conclude with some speculative ideas about how these phenomena might relate to the development of asymmetrical flows. Very strong emission lines have long been known to be characteristic of Mira variables approaching minimum light; most of the modeling results presented at this conference have, however, stressed a variety of absorption features.

Josselin, Plez and Nauron tackled the question of whether the velocity structure in red giant atmospheres can be related directly to the mass loss rate, and whether such a relation, when found, is more likely the result of convective motions or pulsation. They used a set of masks separated by excitation potential to look at the correlation of deep atmospheric velocities with mass loss rates, an approach adumbrated by Willson et al. (1982). The dominant signal is infall, consistent with convection but also with shocks associated with pulsation (Hill \& Willson 1979, Willson et al. 1982). Josselin et al. found that mass loss rates are larger for stars with larger infall velocities - consistent with either more vigorous convection or bigger shocks. They also found that stars with larger radii have larger infall velocities. This latter result is not expected in the case of single mode pulsation: Simple physical arguments suggest that shock amplitudes, and hence infall velocities, should be larger for higher escape velocities for a given pulsation mode (Hill \& Willson 1979) so either pulsation is not the cause of the line shifts or there is a difference in mode (or $Q=P \sqrt{\rho}$ ) within the sample that confounds the trend. Their preferred solution is that the inflow velocities come from convective motions, not pulsation/shocks.

One of the most promising new lines of investigation for extended atmospheres (as in Mira variables) is the use of multi-wavelength narrow bank interferometry. Wittkowski et al. reported on first observations of Miras with the VLTI at ESO. The inescapable conclusion is that none of our favorite simple models will fit the data - not uniform disk, not limb-darkened disk, and not even a Gaussian-like disk. Further, there is some hint of asymmetry in the data.

\section{Winds: How to model? How to measure?}

Stellar winds and mass loss rates are difficult to predict, because the energy going into the wind is typically the small remnant of large amounts. As I have stressed in recent years (Willson 2000 and references therein), Reimers' relation tells us which stars have reached an interesting (fatal) mass loss rate, NOT how individual stars lose mass. Stars with mass loss rates greater than Reimers' relation are therefore rare (and shortlived). This "representative maximum" 
mass loss rate, the Reimers' rate, corresponds to the translation of only about one part in a million of the stellar luminosity into wind luminosity (i.e. GPE and $\mathrm{KE}$ of outflowing material). Thus: A very small fraction of the available energy ends up in the wind. This might lead us to expect that small changes in a model (or in stellar parameters) will lead to big changes in the mass loss rate, and this is indeed what is found. In dust-enhanced winds from pulsating stars, as noted by Woitke in his review, several orders of magnitude in mass loss rate (and an increase by an order of magnitude or more in the outflow velocity) result from adding dust to an otherwise dust-free model. So we expect - and find - that small changes in wind-generation models often have large effects on the mass loss rates, and also that, for a given mechanism and treatment, small changes in the parameters of the stars lead to big changes in the mass loss rates. Woitke's review, discussed in Section I above, covered some of these issues. Here, I have sorted those posters deal with the detection and measurement of the winds by a variety of techniques.

Stellar winds are also hard to measure, because there are typically few species that give a detectable signal from a typical wind (small $\tau$ and/or small volume, hence small emission measure). These species may be badly out of equilibrium, either with excitation $T$ very different from the gas kinetic $T$, or because many of the relevant atoms are tied up in (other) molecules or dust.

Interferometric techniques may be telling us more about stellar winds than about stellar radii, still. I would argue that if the star is not round, or if $r(\lambda)$, then you are not observing the star but rather the wind. However, circular symmetry does not necessarily establish that the stellar diameter is resolved. A better confirmation comes if the diameter is independent of wavelength - but even here one may be fooled if a rising shock front is the site of $\tau=1$ for a wide range of opacity. The result cited by van Belle - that the smallest diameters are found for stars with little or no IR excess - supports the conclusion that what is observed is only rarely the true stellar radius.

In recent years several papers have appeared describing radio continuum observations from masslosing stars. A particularly nice feature is the relatively simple dependence of the opacity on frequency that allows one to map size vs. wavelength and hence constrain the wind structure vs. radial position. Harper and Brown's poster reported on such observations for Betelgeuse, where they resolved it at $6,3.5,2$ and $1.3 \mathrm{~cm}$ and also detected it at $20 \mathrm{~cm}$. They argue for relatively low wind temperatures, do not find evidence for spots or clumps, but do find a strong hint of departure from spherical symmetry, describable to first order as an elliptical shape.

A brave attempt to determine the character of stochastic motions in the winds - to replace "microturblence" with physics - was presented by Böger, Baade and Reimers using GHRS spectra and assuming that the turbulence is described by a Markov process. A correlation length of 0.2 stellar radii gives a good fit to the observations; this is an interesting number since the scale height $(1 / H=-d \ln \rho / d r)$ in a $1 / r^{2}$ wind is $\sim r$. Also of interest: They find that the microturbulence limit for the same parameter configuration deviates considerably from the observed profile, suggesting that wind diagnostics will need to take this into account. 
One of the great puzzles in this field at the moment is the origin of the modulations of winds around protoPNe and some Miras that are roughly if not precisely spherically symmetric and spaced decades to centuries apart - a timescale awkwardly between the dynamic and the thermal time scales for the stellar envelopes. Another issue of considerable importance in the study of AGB mass loss, at least, is the importance of modulation by changes in the stellar properties during the shell flash cycle. The detection of "detached shells" in CO around some Carbon miras (e.g. Olofsson et al.) indicates clearly that in some cases the mass loss rate is far from constant, as expected from shell-flash related luminosity variations.

In a poster, Shöier, Ryde and Olofsson described using CO line and dust continuum emission with far IR lines to set limits on the change in the mass loss rate on a timescale of about a thousand years for three stars. In their poster they described finding that over the last $\sim 10^{4}$ years all three stars have had mass loss rates that are steady (within a factor of 5). In contrast, de Laverny et al. investigated the structure of the shells around this star, finding that all the rings are, in fact, incomplete, not perfectly concentric, and even merging at some position angles. The spacing corresponds to mass loss modulation on scales from 100 years up. Clearly not all the stars are behaving the same way.

One of the more exciting new ways to probe very low mass loss rates was presented by Linsky et al. They found a signature of the collision of the wind in the wing of the Lyman $\alpha$ line, and have used this to estimate mass loss rates from a number of main sequence stars. Their conclusion is that the main sequence mass loss rate scales as $1 /$ time $^{2}$, that the initial mass loss rate for the Sun was probably between 200 and 10,000 times higher than today's, and that a total of up to $3 \%$ of the original mass was probably lost early in the history of the Sun. Finally, they find a correlation of mass loss rate with stellar X-ray flux so one should be able to use X-ray observations at least for large sample statistical studies once this is well-calibrated for main sequence stars. In a companion poster, Wood et al. described the application of this diagnostic in the case of $\alpha$ Tau; the simplest model gave good qualitative agreement but a detailed match remains illusive, perhaps because the ISM is not exactly as assumed or some other detail of the geometry (of the interaction region of the wind with the ISM) is not as simple as was expected.

Nearly all the contributions to this part of the meeting dealt with cool stars, in spite of the broadened label for the session. However, Lobel et al. warmed up the discussion with a poster on the $\mathrm{F}$ type hypergiant $\rho$ Cas. They described an outburst which their spectrum synthesis models gave as yielding a peak mass loss rate of 0.05 solar masses/year with a total loss of 0.03 solar masses over 200 days. Before the outburst, they detected a downflow, giving a hint about what might have triggered the event. There is a movie of this at http://cfa-www.Harvard.edu/ alobel.

\section{Questions remaining}

A What are the best observational constraints on stellar atmospheres? We have depended for a long time on spectra - they are, indeed, informationrich. However, they collapse the spatial information. One of the most 
exciting directions that emerged from the meeting was the variety of ways that we may be able to sort out information about spatial structure radial and non-radial - using new tools including interferometry. Might spectra eventually become a secondary tool, used to confirm deductions coming from more direct methods?

B Why are the current models not better at fitting observations? Are we missing essential physical processes in the dynamical models, and if so, what are they? Or is the difficulty in bridging the gap between what is needed to get good dynamical modeling and what is needed to get good spectrum synthesis?

C Are some of the phenomena we are trying to explain actually extrinsic? Most stars have companions and/or planets; what happens when these are caught in extended atmospheres or winds? A nice variation on this theme is the possible detection of the interaction of the stellar wind with the ISM, observable perhaps via an absorption feature in the wind of Lyman $\alpha$.

On a personal note: The exciting themes of the full meeting, for me, were the dynamics (including convection and pulsation) and advances in spatial resolution. Stellar atmosphere models are no longer placid, well-behaved structure with plane parallel or spherical symmetry and only small departures from equilibrium of all kinds. There is ample opportunity for new work in this field! Werner Weiss and Nik Piskunov have my gratitude for the enormous job of organizing a fine meeting (and their patience with late submissions), Susanne Höfner has my appreciation for efficient co-organization of this session, and last but not least I am very grateful to our four speakers and other contributors for presenting well-considered and interesting papers, making the job of cobbling together this summary relatively easy.

\section{References}

Bowen, G. H. 1988, ApJ, 329, 299

Bowen, G. H., Willson, L. A. 1991, ApJ, 375, L53

Hill, S. J., Willson, L. A. 1979, ApJ, 229, 1029

Kudritzki, R., Puls, J. 2000, ARA\&A, 38, 613

Olofsson, H., Carlstrom, U., Eriksson, K., Gustafsson, B., Willson, L. A. 1990, A\&A, 230, L13

Willson, L. A., Wallerstein, G., Pilachowski, C. A. 1982, MNRAS, 198, 483

Willson, L. A. 2000, ARA\&A, 38, 573 